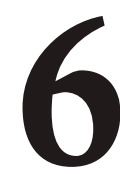

\title{
METÁFORAS DE LA TRANSICIÓN: LA RELACIÓN ENTRE LA ESCUELA INFANTIL Y LA ESCUELA PRIMARIA Y LA PERSPECTIVA DE FUTUROS DOCENTES DE EDUCACIÓN INFANTIL
}

\section{(METAPHORS ABOUT THE TRANSITION: THE RELATIONSHIP BETWEEN PRE-SCHOOL AND PRIMARY SCHOOL AND THE PERSPECTIVES OF FUTURE PRE-SCHOOL TEACHERS)}

Javier Argos González, M M $^{\mathrm{a}}$ Plar Ezquerra Muñoz y Ana Castro Zubizarreta

Universidad de Cantabria

\section{RESUMEN}

La transición entre etapas educativas y, en particular la que se realiza entre la Educación Infantil y la Educación Primaria, resulta un elemento clave y un reto para el adecuado desarrollo y aprendizaje de los alumnos.

En el presente trabajo realizamos un acercamiento a la perspectiva que tienen estudiantes de Magisterio de Educación Infantil en relación con la continuidad entre las referidas etapas, a partir de la experiencia en su primer año de prácticas en centros educativos.

Nuestro análisis se sustenta en las observaciones, reflexiones y argumentaciones efectuadas por los estudiantes en relación con cuestiones que, en torno a la continuidad, les planteamos al iniciar sus prácticas educativas, así como en las metáforas con las que sintetizaban su perspectiva al respecto.

Los resultados constatan una cierta disfunción entre la importancia otorgada a la continuidad en los centros y sus prácticas cotidianas.

De lo anterior se desprende la necesidad de instaurar en los centros propuestas armonizadas que faciliten una adecuada transición entre etapas. 


\section{ABSTRACT}

The transition between educational levels and, in particular, the transition from Pre-School to Primary Education, is a key factor and a challenge for the proper development and correct learning of the students.

In this study we make an enquiry in relation to the perspective that future Early Childhood teachers display regarding the continuity of the aforementioned levels after the experience of their first year of practice in schools.

Our analysis is based in observations, reflections and arguments made by students in relation to questions that they were asked at the beginning of their practices. The main subject of these enquiries dealt with the continuity and the metaphors with which the students summarized their perspective about it.

The results point out that it exists a certain discrepancy between the importance given to continuity in schools and this issue in their daily practices.

It is clear from the above the need to implement harmonized proposals at school easing an adequate transition between levels.

\section{INTRODUCCIÓN}

\subsection{La transición educativa y la necesidad de continuidad entre la Educación Infantil y la Educación Primaria}

El concepto de transición educativa hace referencia al cambio que efectúan los niños desde una fase de la educación a otra, en el que se enfrentan a desafíos desde el punto de vista de las relaciones sociales, el estilo de enseñanza, el ambiente, el espacio, el tiempo, los contextos de aprendizaje y el aprendizaje mismo, haciendo de este proceso algo intenso y con demandas crecientes (Fabian y Dunlop, 2006).

En el tránsito de la etapa de Educación Infantil a la de Educación Primaria el niño necesita ajustarse a un entorno nuevo, muchas veces muy diferente al conocido dentro del ámbito de la Educación Infantil, con un nuevo maestro y nuevos compañeros (Margetts, 2000; Griebel y Niesel, 2000; Fabian, 2000). El comienzo de la escolaridad obligatoria ha sido descrito como uno de los grandes retos a los que el niño se enfrenta en los primeros años de su infancia (Dockett y Perry, 2004), como una transición crítica que puede afectar a su aprendizaje (Featherstone, 2004). 
Por ello, entendemos que es necesario propiciar una continuidad entre ambas etapas que facilite su interacción armónica. Se trataría, como apunta Aguerrondo (2002), de instaurar procesos de articulación, entendiendo que articular supone que la propuesta de enseñanza en un centro educativo guíe sin sobresaltos y en un continuo al alumno para que se desarrolle en todas sus dimensiones.

Esta continuidad puede ser entendida como una necesidad, expresada explícitamente a nivel normativo-prescriptivo en el conjunto de disposiciones que emanan del marco regulador del actual sistema educativo. Así, en la LOE (Art. 3.1) se afirma que "el sistema educativo se organiza en etapas, ciclos, grados, cursos y niveles de enseñanza de forma que asegure la transición entre los mismos y, en su caso, dentro de cada uno de ellos".

La continuidad educativa puede concebirse como un problema, en el sentido de que ésta no es tarea fácil pero, a su vez podemos entenderla como una oportunidad (Gairín, 2005) o, centrándonos en la existente entre la Educación Infantil y la Educación Primaria, como un medio para concebir estas etapas como dos eslabones de un proceso educativo único (Rodríguez y Turón, 2007).

Los numerosos estudios que han abordado este reto pedagógico han señalado los beneficios que, para los niños, comporta esta coordinación. Entre ellos podemos destacar el realizado en EEUU por Kagan, Carroll, Comer y Scott- Little (2006) en cuatro centros de pre-kinderganten y kindergarten en los que se promovía la coordinación, constatando que ésta sólo se realizaba de modo horizontal, siendo casi inexistente la de carácter vertical y que, si los niños experimentan continuidad en su aprendizaje durante las transiciones ésta generará menos dificultades en las nuevas etapas de la escolaridad.

Por su parte, en el ámbito danés, el trabajo de Broström (2002) constata que los niños que han experimentado el contexto de la que será su nueva escuela a través de visitas repetidas o de otro tipo de contactos son más propensos que otros a forjarse expectativas realistas sobre su futuro escolar.

En el estudio realizado en Inglaterra por Bulkeley y Fabian (2006) que analiza la transición efectuada por los niños desde los 5 a los 6 años, recogiendo la perspectiva de maestros, familias y pequeños, se subraya la importancia de una buena comunicación entre todos ellos para favorecer el bienestar emocional del niño. Los trabajos realizados en Australia por Dockett y Perry (2001) y por Margetts (2007) desde la perspectiva del niño, también inciden en que su bienestar emocional y su seguridad se pueden fo- 
mentar si éste conoce las rutinas y las normas de comportamiento de la nueva escuela. Y en la misma línea podemos referir los trabajos de Nielsen y Griebel (2001) y Griebel y Nielsen (2002) que, desarrollados en el ámbito alemán y centrados en la transición del kindergarten a la escuela, afirman que podemos promover el bienestar afectivo y social del niño si le ayudamos en este proceso. Las visitas a la nueva escuela acompañados de la maestra del kindergarten, aún pareciendo que sólo contribuyen a que el niño tenga un conocimiento vago del nuevo colegio, le ayudan a sentirse apoyado y facilitan su adaptación.

\subsection{Algunas constataciones del desencuentro}

Una vez argumentados los beneficios que comporta la transición en general y la desarrollada entre las etapas de Educación Infantil y Educación Primaria en particular, y pensando en algunas pautas que la hagan más armónica, nos parece importante apuntar a continuación algunas prácticas y planteamientos constatados en ambas etapas educativas, fruto de nuestros trabajos con educadores en ejercicio, que, a nuestro entender, dificultan la consecución de una adecuada continuidad.

El primer foco potencial de disonancia estaría vinculado con la orientación o perspectiva pedagógica adoptada en cada etapa, esto es, con las principales metas educativas contempladas en cada una de ellas como más relevantes. En este sentido, se observa que, mientras que la Educación Infantil se plantea desde una perspectiva más global y educativa, en la Educación Primaria se suele transitar hacia una concepción de corte más "academicista”.

Si nos centramos en las estrategias de enseñanza, el rol de los educadores cobra especial protagonismo. Del maestro provocador, sugeridor de propuestas, motivador, compañero o igual, más común en la Educación Infantil, tiende a aparecer en Educación Primaria aquél otro que actúa fundamentalmente como director de la actividad o transmisor de conocimientos.

Los aspectos organizativos (espacios, tiempos, recursos didácticos y formas de agrupamiento de los alumnos) se presentan un tanto diferentes. Así, en la Educación Infantil el espacio considerado como educativo es más global, dinámico y polifuncional que el de la Educación Primaria; la estructura temporal de la actividad suele ser bastante más flexible en la Educación Infantil; los recursos didácticos contemplados en esta etapa son más variados en cuanto a procedencia (editoriales, de desecho, de elaboración propia) y tipo (impresos y manipulativos) que los de la Educación Primaria. 
Por último, los agrupamientos de los niños suelen ser en Educación Infantil más ricos y variados que los habituales en la Educación Primaria.

En estrecha relación con esto último, otro foco de desencuentro sería el relativo a la amplitud interactiva entre los alumnos. Así, mientras que en las aulas de Educación Infantil ésta se potencia y se observa de forma habitual, en las de la etapa de Educación Primaria, por regla general, se vuelve más tenue y menos variada.

En cuanto al papel que se otorga al juego como principio metodológico fundamental en Educación Infantil entendemos, al igual que lo hace Broström (2002), que es el gran perdedor cuando se cambia de etapa. Este autor observa cómo los docentes de Educación Primaria creen que en Educación Infantil “sólo se juega”, devaluando el valor pedagógico de este recurso didáctico. A su vez, comenta la creencia que tienen los maestros de Educación Infantil sobre el mucho tiempo que pasan los niños sentados frente al maestro en Educación Primaria.

También se aprecia desencuentro en la forma en la que desde ambas etapas educativas se presenta y entiende el conocimiento. Así, los planteamientos globalizadores que suelen estar firmemente instaurados en los contextos de Educación Infantil, pierden gran parte de su protagonismo cuando nos adentramos en la etapa de Educación Primaria.

La forma de entender y llevar a cabo la evaluación también se ve más supeditada a los resultados en Educación Primaria, olvidando en ocasiones la necesidad de una evaluación global, amplia y procesual que sí tiene cabida en la Educación Infantil.

Para finalizar, la relación entre la familia y la escuela es un foco de interés a la vez que, en ocasiones, un desencuentro entre ambas etapas. Así, mientras que en la etapa de Educación Infantil la relación con las familias es habitualmente estrecha y preside numerosas actividades, la calidad de las relaciones entre ambos agentes educativos, disminuye conforme nos adentramos en la Educación Primaria.

\section{METODOLOGÍA}

\subsection{Delimitación del objeto de estudio}

En la presente investigación abordamos la temática de la continuidad centrándonos en una perspectiva que, a nuestro entender, puede aportar 
interesantes matices y que, hasta el momento, no ha sido objeto de análisis: la de los futuros docentes de Educación Infantil que se están formando en las aulas universitarias.

Así como en diferentes estudios sobre la continuidad entre las etapas educativas que nos ocupan se ha escuchado la "voz", la perspectiva de las familias, la de los docentes en ejercicio e, incluso la de los propios niños, apenas se ha tenido en cuenta la de los futuros docentes, protagonistas que históricamente han sido más olvidados (Martínez; García y Quintanal, 2006).

Así, al igual que se viene constatando a la hora de estudiar la transición entre etapas, una preocupación creciente por escuchar la voz del niño con el mismo interés que se ha venido haciendo con las familias y educadores (Einarsdóttir, 2007), creemos que sería interesante angular también el estudio desde la perspectiva de los futuros maestros, ya que, en definitiva, se convierten en unos espectadores privilegiados de lo que acontece en la vida de los centros.

El beneficio que comporta un estudio de este tipo puede ser múltiple. De una parte, permite a los futuros docentes y a nosotros, en cuanto que formadores, aproximarnos a la situación vivida en una muestra amplia y significativa de centros escolares y, más en concreto, acercarnos al mundo de las creencias y las prácticas educativas sostenidas por los docentes en ejercicio en relación con la continuidad que, como observaremos a continuación, no siempre son consistentes. Y, por otro lado, este análisis puede ayudar a los estudiantes a reflexionar sobre su pensamiento o conocimiento práctico en relación con esta problemática nuclear en las primeras etapas de la escolarización, ya que, como se pone de manifiesto en algunos estudios (Horppu e Ikonen-Varila, 2004), al iniciar sus estudios de Magisterio pueden tener ya un conocimiento práctico y unas creencias educativas relativamente estables que condicionan su forma de entender la educación.

El objetivo de nuestro estudio, por tanto, se focaliza en conocer la perspectiva que tienen los maestros de Educación Infantil en formación sobre la continuidad educativa entre las etapas de Educación Infantil y Primaria.

\subsection{Diseño y desarrollo del estudio}

El estudio recoge las reflexiones y descripciones efectuadas por futuros maestros de Educación Infantil durante la realización de sus primeras prácticas de enseñanza de seis semanas de duración en unidades de Educación Infantil de la comunidad autónoma de Cantabria. El número total de estudiantes que participaron en el estudio a lo largo de tres cursos académicos 
(2006-2009) fue de 120 y las aulas de Educación Infantil en las que desarrollaron sus prácticas responden a la diversidad de centros existentes, esto es, tanto escuelas infantiles como colegios de Educación Infantil y Primaria, públicos y privados concertados, ubicados en contextos rurales y urbanos.

La demanda que planteábamos a los estudiantes constaba de dos tareas: la primera consistía en plasmar a partir de su primera experiencia de prácticas y de forma descriptiva, su perspectiva sobre la continuidad educativa y la transición entre las etapas de Educación Infantil y de Educación Primaria, articulándose aquélla en torno a tres focos que les planteábamos: la importancia que el contexto escolar se otorgaba a esa coordinación, las diferentes formas de desarrollarla y los aspectos favorecedores y limitadores que creen que conlleva.

Por su parte, la segunda tarea consistía en explicitar, por medio de expresiones metafóricas, el tipo de relación que cada futuro docente observaba entre ambas etapas. Esta tarea nos parecía significativa al entender, al igual que Molina (2002), que las metáforas revelan dimensiones ocultas y profundas convicciones teóricas de los docentes (en formación o en ejercicio), que de manera velada se reflejan en las actividades que desarrollan. Así, la metáfora se convierte en un recurso esencial para explicitar la visión sobre una realidad educativa, en nuestro caso la de la continuidad entre etapas, de una forma sencilla y que permite vincular teoría y práctica pedagógicas.

En el siguiente cuadro sintetizamos las tareas demandadas a los estudiantes:

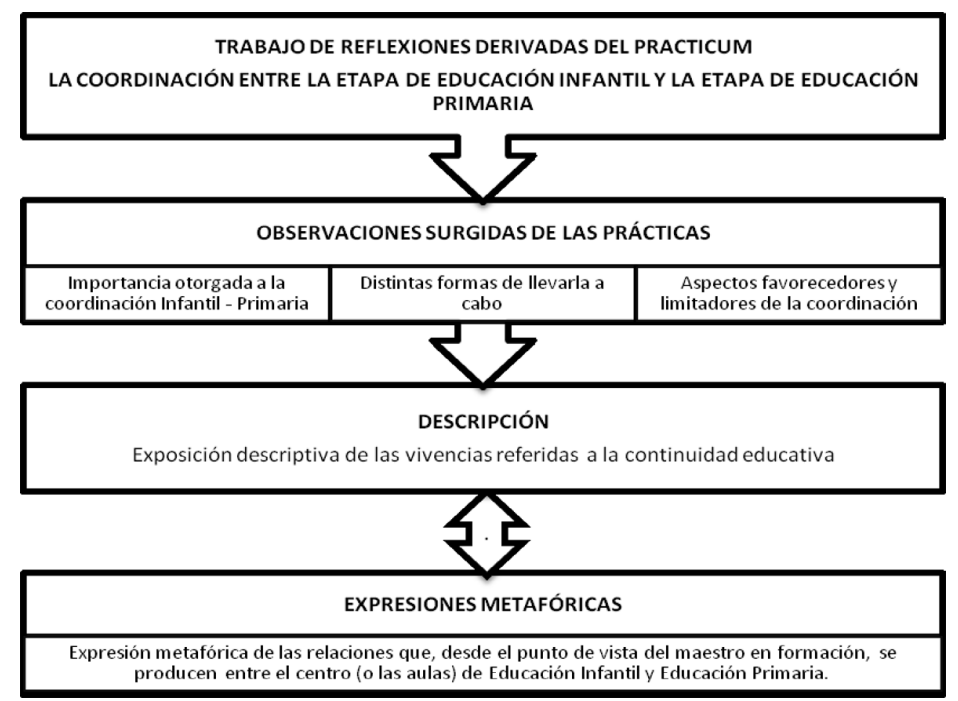


Las descripciones efectuadas por los estudiantes fueron segmentadas en unidades de análisis, es decir, en fragmentos o aspectos recogidos del conjunto de la información obtenida. Estas unidades se analizaron y asignaron a las categorías que planteamos inicialmente en relación con los tres focos de interés anteriormente referidos, identificándose mediante un sistema de codificación que conjugaba diversas variables ${ }^{1}$.

Por lo que respecta a la tarea de acuñar una expresión metafórica constatamos, de una parte, la dificultad que tenían algunos estudiantes para hacerlo correctamente. Por este motivo, tuvimos que desechar aquellas que no respondían al sentido figurado que comporta una expresión de este tipo. Y, de otra parte, observamos los diferentes niveles de amplitud que conllevan. Así, mientras que algunos estudiantes reflejaban en ellas perspectivas globales respecto de la continuidad educativa, otros incidían en aspectos parciales vinculados con alguna de las categorías establecidas.

\section{RESULTADOS}

Presentamos a continuación los resultados obtenidos, generados a partir tanto de las descripciones como de las expresiones metafóricas de los futuros educadores en relación con la continuidad entre las etapas de Educación Infantil y Educación Primaria. Las constataciones y argumentaciones descriptivas y metafóricas se complementan, convirtiéndose ambas en interesantes herramientas interpretativas de las perspectivas mantenidas por los estudiantes.

\subsection{Importancia otorgada a la coordinación entre Educación Infantil y Educación Primaria}

Los futuros educadores constatan la importancia que, al menos verbalmente, otorgan los maestros en ejercicio a la continuidad educativa, expresando éstos la necesidad de prestar especial atención a aquellos momentos que supongan la incorporación a nuevas situaciones dentro del sistema educativo y, en concreto, a todas aquellas que implican un cambio de etapa educativa y/o un cambio de centro o de grupo de compañeros. Así, se observa un consenso al otorgar una gran importancia a la coordinación y relación entre etapas y en la necesidad de prever mecanismos que faciliten la continuidad, de forma que los alumnos vivencien este proceso como algo progresivo y armónico.

Se da una importancia muy elevada a la coordinación entre educación infantil y primaria, ya que lo que se pretende es una formación integral del 
niño y se considera que esto sólo va a ser posible a través de una buena coordinación de todo el centro y una actuación conjunta (46, BbII).

Gracias a esta coordinación el alumno puede pasar al ciclo de primaria de una forma más suave, es decir, casi sin notar cambio (90, AbII).

En el centro se otorga gran importancia a la relación entre etapas. Si buscamos una educación infantil de calidad, tenemos que tener en cuenta no sólo aquello que debemos enseñar en ella, sino que todas estas cosas que ofrecemos a los niños de la etapa de 0 a 6 años, tengan su continuidad en la siguiente etapa, la educación primaria de 6 a 12 años (34, BbII).

Pero, a pesar de este consenso inicial sobre la importancia de la coordinación entre estas etapas y sobre su importante papel en cuanto que factor de calidad educativa y de recurso facilitador de las transiciones vividas por los niños, lo constatado por bastantes de los futuros docentes hace pensar que existen incoherencias entre el pensamiento y la acción de los docentes en ejercicio acerca de la referida relación entre etapas. Un ejemplo de ello es lo apuntado a continuación por uno de nuestros estudiantes:

Es importante la transición entre etapas, pero no puedo comentar gran cosa ya que si existe relación entre las dos etapas, la desconozco, pues no la he presenciado de ninguna manera durante mi estancia en el centro (38, AbII).

Al analizar el tipo de vínculo que se establece entre la etapa de Educación Infantil y la de Primaria, los estudiantes observan la existencia de una relación de tipo jerárquico en la que la etapa de Educación Primaria goza de una posición de poder que marca contenidos y metodología al último curso de Educación Infantil. Podemos decir, que según la percepción de los futuros docentes, la etapa de Educación Infantil se somete a las exigencias de la de Educación Primaria, llegando a trasladar la estructura, los objetivos y la metodología de ésta, trayendo como consecuencia una cierta "primarización" de la Educación Infantil:

La gran mayoría de las actividades que se realizan en la etapa de infantil van dirigidas o están enfocadas para que cuando el alumno acceda a primaria tenga una buena base corriendo el riesgo de que se pierda un poco esa perspectiva de educación infantil. Una de las mayores preocupaciones de las tutoras de infantil es conseguir que los niños estén bien preparados para cuando salten a la nueva etapa de primaria (26, BaIII).

La etapa de Educación Infantil presenta, en ocasiones, una identidad difuminada por los intereses y las demandas de la etapa posterior. Así, algún estudiante contempla este hecho en sus reflexiones: 
Alguna profesora del primer ciclo de primaria, concretamente del $1^{\circ}$ curso, se ha quejado del rendimiento de alguno de sus alumnos y ha comentado que ha tenido que ir más lenta a la hora de realizar las actividades de lectura y escritura (100, BbII).

Aunque a nivel legislativo parece fuera de toda duda la consolidación de la etapa de Educación Infantil como etapa con identidad propia, la práctica cotidiana se perfila y hace vislumbrar, en ocasiones, una situación diferente. Así, en el siguiente fragmento, una estudiante explicita la lucha de una de las maestras por evitar la anticipación de contenidos de corte academicista en las aulas de Educación Infantil.

Yo escuché esta frase de mi tutora: Ya no es preescolar, es educación infantil, así que no tengo que preparar a los niños para primaria (19, AbII).

\subsection{Distintas formas de llevar a cabo la coordinación entre las etapas}

Desde la perspectiva de los futuros docentes, el tipo de coordinación predominante en los centros escolares es de carácter esporádico, que se reduce, generalmente, a una reunión anual o a encuentros puntuales a lo largo del curso. Ello suele comportar una relación un tanto empobrecida, motivada por la falta de contacto, de intercambio y de trabajo en común, que se limita con frecuencia a un trasvase de información sobre los pequeños al finalizar éstos la etapa de Educación Infantil, o a la participación en fiestas y en otros eventos ocasionales que se desarrollan en el centro escolar. Nuestros estudiantes manifiestan de forma recurrente reflexiones como las siguientes:

Respecto a las relaciones personales entre los distintos ciclos y niveles, se reducía principalmente a momentos concretos como son las celebraciones o determinadas actividades (112, AbIII).

Los docentes de infantil sólo hablan y se reúnen con los docentes de primaria cuando los niños están en último curso de Educación Infantil y van a pasar al siguiente ciclo. Hasta entonces, la relación es inexistente (32, AbII).

Esta relación un tanto esporádica y superficial es, tal vez, la causante de que algunos maestros en ejercicio la perciban como poco necesaria, propiciándose, de este modo, una especie de círculo vicioso en el que la escasez de tiempo con la que cuentan los docentes puede generar el dedicar poco a la comunicación entre los docentes de ambas etapas. Un ejemplo de esta situación, es el observado por un estudiante en el apunte siguiente: 
Se suelen establecer una serie de reuniones entre la coordinadora de infantil y la de primaria, pero me han comentado, que en muchas ocasiones no se llevan a cabo por falta de tiempo (80, AbII).

Ante esta situación, la mejora de esa relación requeriría de los docentes el transitar desde actitudes un tanto estáticas y conformistas a otras que promueven la iniciativa y la búsqueda de soluciones. En esta difícil tarea, algunos estudiantes perciben que el trabajo por proyectos y el trabajo en equipo de los docentes, favorecen la coordinación entre etapas, haciendo que ésta sea estable y continuada.

Recogemos a continuación la perspectiva de diferentes estudiantes que han tenido la oportunidad de vivenciar situaciones en las que se facilita la continuidad, gracias a los proyectos comunes en los que participan los maestros de Educación Infantil y del primer ciclo de Educación Primaria:

La relación existente entre ambas etapas en este centro es muy intensa. Compartían entre las etapas el mismo tema a desarrollar. En las reuniones del ciclo de infantil, en muchas ocasiones se sumaban las maestras de $1^{\circ}$ y $2^{\circ}$ de primaria para planificar entre todos cómo se va a desarrollar las distintas actividades de cada uno de los temas. El año escolar en este centro se dividía en tres grandes proyectos... (59, AbII).

Para conseguir este trabajo común entre diferentes cursos ha sido necesario que el equipo docente de infantil y el correspondiente al primer ciclo de primaria formen un único grupo que trabajen a favor de estos proyectos diseñados con el objetivo de unir ambas etapas. De esta manera diluyen las diferencias existentes y hacen desaparecer el temor al cambio (21, AaII).

\subsection{Aspectos favorecedores y limitadores de la coordinación}

Analizando los aspectos que, desde la perspectiva de los estudiantes, favorecen la coordinación deberíamos de aludir, en primer lugar, a la existencia de un ambiente familiar en el centro. La creación de éste, en muchas ocasiones, se consigue como consecuencia del tamaño reducido de la escuela:

Que el centro sea pequeño favorece la creación de un ambiente familiar, pues todos se conocen y es más fácil crear lazos afectivos. Al ser pocos profesores, el trabajo en equipo y la coordinación entre las diferentes etapas es más sencillo. El ambiente familiar y la armonía que existe entre todos los miembros de la comunidad educativa también facilitan la coordinación y la cooperación. Pues cuando existe esta armonía entre todos los miembros es más fácil debatir y ponerse de acuerdo sin que surjan enfrentamientos (8, BaIII). 
Como se apreciará en la reflexión anterior de un estudiante, un contexto favorecedor de la continuidad entre etapas se consigue también mediante la consecución de un ambiente no ya sólo familiar sino, además, armónico. En este mismo sentido se expresan algunos otros futuros docentes:

A parte de las reuniones marcadas por el centro, las profesoras de ambas etapas tenían una buena relación por lo que había un buen clima de trabajo, unos no se pisaban las tareas de los otros sino que se ayudaban entre ellos (37, AbII).

La coordinación entre las etapas es total, tanto en espacios como en tiempos, recursos, profesores y contenidos. La colaboración entre la etapa de primaria y la de infantil es doble: por un lado, los alumnos más mayores participaban muchas veces en la organización y cuidado de las actividades de los niños más pequeños, sobre todo en días como el de la celebración del colegio, el día de la Paz, excursiones...por otro lado, los profesores se ayudan mutuamente, si en algún momento uno está especialmente agobiado... (1, B aIII).

Por último, entre los aspectos facilitadores de la coordinación constatamos como importante la cercanía espacial entre las unidades de ambas etapas educativas y en las situaciones en las que los niños comparten espacios comunes del centro:

Las aulas de 5 años y $1^{\circ}$ y $2^{\circ}$ de Primaria se encuentran en el mismo pasillo para que los niños vayan familiarizándose con la etapa de los mayores (11, BbII).

Se potencian actividades en el recreo para favorecer la comunicación entre los niños de infantil y primaria (24, BaIII).

Un contexto un tanto especial en el que la continuidad educativa entre etapas es algo consustancial a la práctica pedagógica, es el de las escasas, pero aún existentes, escuelas unitarias. En ellas la continuidad forma parte de la cotidianeidad relacional e impregna los intercambios de todo tipo que niños y niñas de diferentes edades, niveles y etapas establecen. En este sentido, presentamos la reflexión que realiza una alumna:

Al ser una escuela unitaria los niños y niñas de infantil y primaria estaban juntos todas las horas lectivas. Además por la metodología que impartían se observaba cómo los mayores ayudaban a los pequeños en todo lo que necesitaban (4, BbII).

$\mathrm{Al}$ analizar los aspectos limitadores de la continuidad entre ambas etapas, los futuros docentes atribuyen a diferentes tipos de motivos la, en ocasiones constatada, insuficiencia relacional. 
En primer lugar, creen que el entorno escolar tiene una importancia relevante en este proceso de continuidad y tienden a vincularla con las características fijas de los espacios y, en concreto, con los edificios escolares que, como acabamos de señalar, pueden contribuir positivamente al referido proceso. Los estudiantes parten de la creencia de que "a mayor proximidad física entre las etapas, mayor relación entre las mismas”. Por ello, piensan que un centro escolar conformado por edificios diferentes, destinados cada uno a una etapa, potencia el aislamiento y la falta de comunicación.

A raíz de que estos edificios están separados, a simple vista parece que funcionan como centros individuales, ya que los alumnos de infantil y de primaria nunca mantienen contacto físico, entrando al recinto escolar por puertas distintas y teniendo patios propios (8, BbII).

Debido a esta distribución del colegio, los maestros apenas se veían a lo largo del día o incluso a lo largo de las semanas (17, BaIII).

La falta de coordinación entre etapas era debida quizá a que las aulas se encontraban ubicadas en distintos pasillos y los profesores apenas llegaban a relacionarse diariamente. Esto era una queja frecuente de las profesoras de infantil: nunca nos enteramos de nada, nos tienen abandonados (33, AbII).

Otra opción de configuración y distribución espacial sería aquella en la que ambas etapas comparten un mismo edificio. En estos casos, aún cumpliéndose a priori el criterio de proximidad establecido por los estudiantes como facilitador de la continuidad entre etapas, contrasta con algunas de sus constataciones sobre el desarrollo de la práctica educativa, en la que vuelven a señalar problemas de comunicación y coordinación. Para ellos, las causas que interfieren en la continuidad educativa una vez encontrada una mejora de las condiciones del entorno escolar, tienden a vincularlas con los tiempos escolares.

Así, en sus reflexiones plantean que las etapas educativas, aún compartiendo edificio y espacios escolares (patios, sala de profesores, aulas para didácticas especiales), la existencia de tiempos de uso claramente diferenciado tiende a producir un efecto de "invisibilidad" tanto en niños como en educadores, que queda reflejado en la constatación de un estudiante que expresa la dificultad que tiene para coincidir con un compañero que realiza el Practicum en la etapa de Educación Primaria del mismo centro escolar: "estamos, pero no nos vemos".

La planificación exhaustiva de tiempos de uso diferenciado de las instalaciones y servicios escolares suele responder al objetivo prefijado de no coincidir, tal y como ilustra un estudiante con su reflexión: 
En cuanto a las horas del recreo, había una hora para los alumnos de infantil, otra para los de primaria y otra para los de secundaria y bachiller. En cuanto a la utilización de las aulas, como por ejemplo gimnasio, el aula de música o el aula de informática, existían también diferentes horarios para su uso, esto permitía que todas las etapas no coincidiesen (10, BaIII).

Otra posible causa del desencuentro identificada por nuestros estudiantes es la exaltación de las características propias de cada etapa por parte de los maestros en ejercicio, así como sus hábitos y prácticas profesionales, a la vez que un sentimiento de autosuficiencia del profesorado de cada etapa que limita la posibilidad de emprender procesos de reflexión conjunta. Los alumnos subrayan la existencia de grandes diferencias pedagógicas entre una etapa y otra tal y como expresan varios estudiantes en los siguientes comentarios:

Pese a estar en el mismo colegio la educación infantil y la primaria, no había la coordinación que se desea. Empezando por la metodología y la forma de trabajar; mientras que en infantil se trabaja por proyectos que engloban las distintas áreas de aprendizaje y se procura que sea el niño quien lleve a cabo su aprendizaje, en primaria nada de esto se lleva a cabo (78, BbII).

Cada día veía más diferencias en la forma de trabajar en las aulas de infantil y en las de primaria, pasando de trabajar por proyectos, de forma activa, participativa y flexible... a un método estático donde se limitan a los libros de texto (14, BbII).

\subsection{Las expresiones metafóricas como síntesis de las percep- ciones de los estudiantes sobre la transición}

En la siguiente tabla recogemos algunos ejemplos representativos de las metáforas acuñadas por los estudiantes. Se puede observar que, aunque mayoritariamente responden a la percepción de los estudiantes sobre la realidad vivida en los centros, en algunos casos la utilizan como una herramienta para manifestar la forma en la que creen que esa relación debería producirse.

En algunos casos ofrecen una perspectiva positiva acerca de la transición, mientras que en otros, presentan un carácter más negativo o limitador. El carácter que asignamos a cada una de ellas responde a la argumentación elaborada por cada estudiante en el marco de sus reflexiones sobre las tres cuestiones planteadas y, por ello, no se podría entender adecuadamente sin tener en cuenta dicho contexto. 


\begin{tabular}{|c|c|}
\hline METÁFORA & TIPO DE RELACIÓN \\
\hline La distancia que hay entre los dos extremos de un puente. & Positiva \\
\hline El paso de un baby de colores a una bata blanca. & Negativa \\
\hline $\begin{array}{l}\text { Dos desconocidos en un ascensor, se habla del tiempo, se } \\
\text { mira el reloj y te bajas en tu piso. }\end{array}$ & Negativa \\
\hline $\begin{array}{l}\text { Es el pasar de "mamá voy al cole a jugar" a "mamá no } \\
\text { quiero ir al cole". }\end{array}$ & Negativa \\
\hline $\begin{array}{l}\text { La educación infantil es el cimiento de un edificio que se } \\
\text { construirá en las etapas educativas posteriores. }\end{array}$ & Positiva \\
\hline Una pareja de divorciados, lo justo y necesario. & Negativa \\
\hline $\begin{array}{l}\text { Un debate electoral. Cada candidato defiende sus ideas y se } \\
\text { ciñe a su discurso. }\end{array}$ & Negativa \\
\hline $\begin{array}{l}\text { Un tren encarrilado y comprometido entre el punto de } \\
\text { partida y la llegada a la estación. }\end{array}$ & Positiva \\
\hline $\begin{array}{l}\text { Si a la cebra le borran las rayas puede acabar convirtién- } \\
\text { dose en un burro. }\end{array}$ & Negativa \\
\hline $\begin{array}{l}\text { Dos ríos con distintos afluentes que van a desembocar en } \\
\text { el mismo mar }\end{array}$ & Positiva \\
\hline $\begin{array}{l}\text { Pasar de la infancia a la vejez pero sin apenas experiencias } \\
\text { intermedias. }\end{array}$ & Negativa \\
\hline De infantil a primaria, del pájaro libre al pájaro enjaulado. & Negativa \\
\hline Infantil y primaria, dos hermanas de la mano. & Positiva \\
\hline Infantil y primaria, dos eslabones de una misma cadena & Positiva \\
\hline Infantil es un soldado raso y primaria su sargento. & Negativa \\
\hline Infantil y primaria, un teléfono escacharrado. & Negativa \\
\hline Infantil y primaria, dos islas perdidas. & Negativa \\
\hline $\begin{array}{l}\text { De infantil a primaria, de la fotocopia en color a la fotoco- } \\
\text { pia en blanco y negro. }\end{array}$ & Negativa \\
\hline $\begin{array}{l}\text { Dos personas que hablan idiomas diferentes. Se necesita } \\
\text { un intérprete que les ayude a entenderse. }\end{array}$ & Negativa \\
\hline
\end{tabular}




\begin{tabular}{|c|c|}
\hline $\begin{array}{l}\text { Las piezas de ajedrez, cada una tiene su función pero todas } \\
\text { son imprescindibles. }\end{array}$ & Positiva \\
\hline $\begin{array}{l}\text { Los miembros de una orquesta, es necesario saber llevar el } \\
\text { compás para no desafinar. }\end{array}$ & Positiva-deseable \\
\hline $\begin{array}{l}\text { Encender un fuego, crearlo cuesta pero si se consigue, la lla- } \\
\text { ma puede permanecer encendida durante mucho tiempo. }\end{array}$ & Positiva-deseable \\
\hline $\begin{array}{l}\text { La suma que resta: pasar de Infantil a primaria es un gran } \\
\text { logro para el desarrollo del niño pero, también, puede ser } \\
\text { una desventaja para dimensiones como la creatividad, la } \\
\text { espontaneidad y las emociones que pierden protagonismo } \\
\text { en la etapa Primaria. }\end{array}$ & Dual \\
\hline $\begin{array}{l}\text { El agua. Es vital para la vida pero puede manifestarse de } \\
\text { diferentes maneras, líquida, congelada, vaporizada. }\end{array}$ & Diversa \\
\hline Infantil y Primaria, son las dos patas de un mismo banco & Positiva \\
\hline $\begin{array}{l}\text { Infantil y Primaria son las letras que unidas forman pala- } \\
\text { bras con sentido. }\end{array}$ & Positiva \\
\hline $\begin{array}{l}\text { Infantil y Primaria son territorios inexplorados para los } \\
\text { profesores de cada etapa. }\end{array}$ & Negativa \\
\hline $\begin{array}{l}\text { Infantil y Primaria son como el aceite y el vinagre, uno no } \\
\text { se concibe sin el otro para aliñar una ensalada. }\end{array}$ & Positiva \\
\hline $\begin{array}{l}\text { Los órganos del cuerpo humano, cada uno tiene una fun- } \\
\text { ción y su presencia es vital. }\end{array}$ & Positiva \\
\hline Infantil y Primaria son el agua y la luz para una planta. & Positiva \\
\hline Fría como el hielo. & Negativa \\
\hline Primaria es la tierra e Infantil su satélite. & Negativa \\
\hline $\begin{array}{l}\text { La relación entre Infantil y Primaria es como un diamante } \\
\text { en bruto, tiene mucho valor, pero en mi centro hay que pu- } \\
\text { lirla mucho. }\end{array}$ & Negativa-dilemática \\
\hline
\end{tabular}

\section{DISCUSIÓN}

La perspectiva positiva que transmiten los docentes de Educación Infantil a los estudiantes en relación con la importancia que ha de concederse a la continuidad educativa y a la coordinación que ha de propiciarse entre etapas para facilitarla, es un elemento de partida importante aunque, también hemos de señalar, que se pueden observar algunas incoherencias entre su pensamiento y su práctica educativa. 
Los estudiantes ponen de manifiesto que los maestros en ejercicio no siempre contemplan el carácter propio y la identidad de la etapa de Educación Infantil como cabría esperar desde la consolidación que ésta ha tenido en las últimas décadas. Parece que la relación de esta etapa con la de Educación Primaria, en ocasiones, en lugar de establecerse desde las peculiaridades de cada una de ellas, está condicionada por las características y pretensiones de esta última.

La coordinación esporádica que, con carácter recurrente, perciben nuestros estudiantes entre las dos etapas educativas referidas, a nuestro entender, debería dar paso a otra más sistemática, regular y estable, integrándose en el quehacer pedagógico cotidiano. Así se facilitaría una relación más fluida entre ambas etapas, que podría conllevar la implicación en proyectos y en actividades compartidos entre el conjunto de niños y educadores. Somos conscientes de que el aumento de la burocratización de la enseñanza demanda cada vez más tiempo a los profesionales impidiéndoles prestar la atención que merecerían a otras cuestiones importantes como las relativas a las transiciones educativas.

Los estudiantes perciben la existencia de diferencias pedagógicas entre ambas etapas educativas más allá de las consustanciales a la identidad de cada una. Parece que los docentes de ambas mantienen una cierta percepción de autosuficiencia al considerar que cada uno conoce bien su tarea y, consiguientemente, que no precisan emprender procesos de reflexión y de aprendizaje conjuntos. En este mismo sentido se manifiesta Gairín (2002) cuando hace referencia a una cultura y forma de hacer individualista. Entendemos que el trabajo en equipo es el medio idóneo para potenciar la continuidad educativa, aunque no podemos olvidar que trabajar en grupo no es tarea fácil y que algunos profesionales siguen primando el trabajo de tipo individual.

A la luz de las observaciones realizadas por los estudiantes, se constata la existencia de diferentes variables, tanto facilitadoras como limitadoras, de la continuidad. Así, la presencia o ausencia de planteamientos conjuntos de carácter tanto organizativo como metodológico, puede convertirse en un elemento condicionante del desarrollo de los procesos de continuidad.

La perspectiva que ofrecen los estudiantes, tanto en sus descripciones como a través del uso de las herramientas metafóricas con las que intentan reflejar cómo perciben la continuidad entre etapas, es de un carácter más bien negativo, aunque también son optimistas manifestando que es algo que se puede mejorar. Entendemos que el haber enfrentado a los futuros docentes ante esta problemática, lejos de percibirlo como un impedimento en su formación, hemos de considerarlo como una oportunidad para ha- 
cerles conscientes de la necesidad de buscar alternativas de mejora de la escuela. Este quizá sea uno de los grandes retos que deban contemplarse de cara a dotar de coherencia a las propuestas pedagógicas de los centros escolares. 


\section{NOTAS}

1. Codificación:Número de caso, Centro educativo ubicado en entorno rural (A), Centro educativo ubicado en entorno urbano (B), Centro educativo privado concertado (a), Centro educativo público (b), Escuela de Educación Infantil (I), Centro escolar de Educación Infantil y Primaria (II), Centro de Educación Infantil, Primaria y Secundaria (III). 


\section{REFERENCIAS BIBLIOGRÁFICAS}

Aguerrondo, I. y otros (2002). La Escuela del Futuro III: Qué hacen las escuelas que innovan. Buenos Aires: Papers Editores.

Broström, S. (2002).Communication \& Continuity in the transition from kindergarten to school, in Fabian, $\mathrm{H}$. and Dunlop, A.-W. (Ed.) Transitions in the Early Years. Debating continuity and progression for children in early education. London: RoutledgeFalmer, 52 -64 .

Bulkeley, J. and Fabian, H. (2006). Wellbeing and Belonging during Early Educational Transitions. International Journal of Transitions in Childhood, 2, 18-30.

Dockett, S. and Perry, B. (2001). Starting school: effective transitions. Early Childhood Research and Practice, 3 (2), 1-18.

Dockett, S. and Perry, B. (2004). Starting school: Perspectives of Australian children, parents and educators. Journal of Early Childhood Research, 2 (2), 171189.

Einarsdóttir, J. (2007). Children's voices on the transition from preschool to primary school, in Dunlop, A-W. and Fabian, H. (Eds). Informing transitions in the early years. Research, policy and practice. Berkshire, UK: Open University Press/McGraw Hill Education, 7492.

Fabian, H. (2000). Empowering children for transitions. Paper presented at the EECERA 10 $0^{\text {th }}$ European Conference on Quality in Early Childhood Education, London.

Fabian, H. and Dunlop, A. W. (2006). Outcomes of Good Practice in Transi- tion Processes for Children Entering Primary School. Paper Commissioned for the EFA Global Monitoring Report 2007, Strong Foundations: Early Childhood Care and Education. Paris: UNESCO.

Featherstone, S. (2004). Smooth Transitions. Nursery World, 27 May, 104 (3919), 14-15.

Gairín, J. (2002). Estrategias colaborativas para la mejora organizativa de la escuela rural, en Lorenzo, M. y otros (coord.) Liderazgo educativo y escuela rural. Granada: Grupo Editorial Universitario, 125-153.

Gairín, J. (2005). El reto de la transición entre etapas educativas. Aula de Innovación Educativa, 142, 12-17.

Griebel, W. and Niesel, R. (2000). The children's voice in the complex transition into kindergarten and school. Paper presented at the 10th Annual Conference of the European Early Childhood Educational Research Association, London, UK.

Griebel, W. and Niesel, R. (2002). Coconstructing transition into kindergarten and school, in Fabian, H. and Dunlop, A-W. (eds.)Transitions in the Early Years: Debating continuity and progression for children in early education. London, UK: Routledge Falmer, $64-76$.

Horppu, R. and Ikonen-Varila, M. (2004). Mental models of attachment as a part of kindergarten student teachers' practical knowledge about caregiving. International Journal of Early Years Education, 12 (3), 231-243.

Kagan, S.L., Carroll, J., Comer, J. and Scott-Little, C. (2006). Alignment: A 
missing link in early childhood transitions? Young Children, 61 (5), 26-32.

Margetts, K. (2000). Indicators of children's adjustment to the first year of schooling. Journal of Australian Research in Early Childhood Education, 7 (1), 20-30.

Margetts, K. (2007). Preparing children for school - benefits and privileges. Australian Journal of Early Childhood, 32 (2), 43-50.

Martínez, M. M., García, B. y Quintanal, J. (2006). El perfil del profesor universitario de calidad desde la perspectiva del alumnado. Revista Educación XX1, 9, 183-198.
Molina, M. (2002). Las educadoras y sus metáforas educativas: dos investigadores en acción. Cuaderno de Investigación en la Educación, 17, 1-16.

Niesel, R. and Griebel, W. (2001). Transition to schoolchild. What children tell about school and what they teach us. Paper presented at the 11th European Early Childhood Education Research Association Conference, Alkmaar, Netherlands.

Rodríguez, A. C. y Turón, C. A. (2007). Articulación preescolar-primaria: recomendaciones al maestro. Revista Iberoamericana de Educación, 44 (4), 1-6. 


\section{PALABRAS CLAVE}

Transición, educación infantil, educación primaria, continuidad educativa, docentes en formación inicial

\section{KEY WORDS}

Transition, early childhood education, primary education, educational continuity, preservice education

\section{PERFIL ACADÉMICO Y PROFESIONAL DE LOS AUTORES}

Javier Argos González, catedrático de Escuela Universitaria del área de Teoría e Historia de la Educación en el Departamento de Educación de la Universidad de Cantabria. Ostenta la Vicepresidencia del Consejo Escolar de Cantabria. Es profesor-tutor del Centro Asociado de la UNED de Cantabria. Sus principales líneas de investigación se vinculan con el ámbito de la Educación Infantil en el que cuenta con publicaciones nacionales e internacionales.

$\mathrm{M}^{\mathrm{a}}$ Pilar Ezquerra Muñoz, profesora Titular de Universidad del área de Teoría e Historia de la Educación en el Departamento de Educación de la Universidad de Cantabria. Su investigación se enmarca en el ámbito de la Educación Infantil, en el que ha publicado diferentes trabajos tanto a nivel nacional como internacional.

Ana Castro Zubizarreta, profesora ayudante del área de Teoría e Historia de la Educación de la Universidad de Cantabria. Doctora por la Universidad de Deusto. Su trabajo docente e investigador está vinculado con el ámbito de la Educación Infantil.

Dirección de los autores: Departamento de Educación Universidad de Cantabria Avda. Los Castros, s/n 39005 Santander (Cantabria)

e-mail: argosj@unican.es ezquermp@unican.es castroza@unican.es

Fecha de Recepción del Artículo: 30. Diciembre. 2009

Fecha de revisión del artículo: 22. Mayo. 2010

Fecha de Aceptación del Artículo: 29. Septiembre. 2010 